Revista de Matemática: Teoría y Aplicaciones 4(2): 43-50 (1997)

\title{
PRECUANTIZACIÓN EN DIFEOLOGÍAS SUAVES
}

\author{
Carlos A. Torre ${ }^{1}$
}

\begin{abstract}
Resumen
Definimos una precuantización en la categoría de espacios difeológicos suaves (una categoría que incluye sistemas de dimensión finita e infinita), en el caso en que la forma simpléctica es exacta; extendiendo, para este caso, los resultados de Kirillov, Kostant y Souriau que son desarrollados para sistemas físicos de dimensión finita.
\end{abstract}

\begin{abstract}
We define a prequantization in the category of smooth diffeological spaces (a category which includes finite and infinite dimensional systems), in the case where the symplectic form is exact, extending, for this case, the results of Kirillov, Kostant and Souriau which aredeveloped for finite dimensional physical systems.
\end{abstract}

\section{Introducción}

Los grupos de simetría y las variedades simplécticas (las cuales se relacionan con los primeros a través de las órbitas coadjuntas [6]), juegan un papel central en el estudio de sistemas físicos, tanto de dimensión finita como infinita [1,10], tanto en la física clásica como en la cuántica y en la relación entre ambas. Los modelos para el caso de dimensión finita se encuentran ampliamente desarrollados, sin embargo, el caso de dimensión infinita, aunque de igual importancia, no se ha logrado desarrollar satisfactoriamente.

La categoría de espacios difeológicos fué construida por Souriau [7] y ha sido desarrollada por Donato [2,3] e Iglesias [4,5] con el objeto de estudiar sistemas físicos de dimensión infinita. Permitiendo la definición de muchos objetos geométricos tales como formas, homotopías, fibraciones, y permite una precuantización cuando cierta obstrucción cohomológica es cero. Sin embargo esta teoría no permite extender, en el contexto de espacios difeológicos muchos resultados ampliamente desarrollados en el caso de dimensión finita. Así por ejemplo, la definición de formas es covariante, sin utilizar campos vectoriales, puesto que no se cuenta con un fibrado tangente. La precuantización también es obtenida sin una descripción a través de un álgebra de Poisson, las cuales son útiles para describir la evolución de un sistema físico clásico.

Con el objeto de resolver estas dificultades hemos definido en [9] los espacios difeológicos suaves, que incluyen los sistemas de dimensión finita, así como subgrupos de difeomorfismos y órbitas coadjuntas de dimensión infinita (éstas son variedades simplécticas

\footnotetext{
${ }^{1}$ Escuela de Matemática, Universidad de Costa Rica, 2060 San José, Costa Rica
} 
[8]). En estos modelos se pueden construir fibrados tangentes que permiten la definición de álgebras de Lie de campos vectoriales, formas diferenciales y una cohomología de De Rham.

En este artículo definimos en la sección 2 las categorías de difeologías y difeologías suaves. En la sección 3 se define una difeología suave para los fibrados tangente, los campos vectoriales, las formas diferenciales y la cohomología de De Rham. En la sección 4 definimos las difeologías simplécticas, así como un álgebra de campos vectoriales hamiltonianos y un álgebra de Poisson en el contexto general de espacios difeológicos suaves. Finalmente se construye una precuantización en el caso en que la forma simpléctica es exacta, de esta forma extendiendo los resultados conocidos en el caso de una variedad de dimensión finita $[10]$.

\section{Difeologías suaves}

Dado un conjunto $S$, una n-placa de $S$ es una función $p: U \rightarrow S$ donde $U$ es un abierto de $\mathbf{R}^{n}$. Un conjunto de placas $P(S)$ forma una difeología si

(i) Las imágenes de las placas cubren $S$.

(ii) Si un conjunto $\left(p_{i}\right)$ de n-placas admite una extensión común, entonces la más pequeña de tales extensiones es una n-placa en $P(S)$.

(iii) Si $\phi \in C^{\infty}\left(U^{\prime}, U\right)$ donde $U^{\prime} \subset \mathbf{R}^{n}$ es abierto y $p$ : $U \rightarrow S$ entonces $p \circ \phi \in P(S)$.

El conjunto de difeologías de un conjunto $S$ se ordena bajo la relación $\mathcal{D}$ menos fina que $\mathcal{D}^{\prime}$ si $\mathcal{D} \subset \mathcal{D}^{\prime}$. La difeología formada por las placas localmente constantes (llamada discreta) es la menos fina.

Todo conjunto de placas $R(S)$ que recubre un espacio $S$ genera una única difeología llamada la difeología generada por $R(S)$ definida como la intersección de todas las difeologías que contienen a $R(S)$.

Dados dos espacios $R, S$ con difeologías $P(R), P(S)$ respectivamente, una función $f: R \rightarrow S$ es llamada diferenciable si para todo $p \in P(R)$ se tiene que $f \circ p \in P(Y)$. Denotemos por $C^{\infty}(R, S)$ al conjunto de tales funciones. $f$ se llama un difeomorfismo si $f$ es biyectiva y $f^{-1}$ es también diferenciable.

Dado $f: S \rightarrow S^{\prime}$, si $P(S)$ es una difeología de $S$, se llama difeología imágen de $S^{\prime}$ a la difeología menos fina $P^{f}\left(S^{\prime}\right)$ tal que $f$ es diferenciable. Toda placa $p \in P^{f}\left(S^{\prime}\right)$ tiene la forma $f \circ \alpha$, con $\alpha \in P(S)$, para algún abierto $U_{r}$ de cada punto $r$ del dominio de $p$. Por ejemplo, para un cociente $S / \sim$ de $S$ podemos construir la difeología cociente a través de la proyección canónica.

Si ahora $P\left(S^{\prime}\right)$ es una difeología de $S^{\prime}$, la difeología $P_{f}(S)$ es la difeología más fina tal que $f$ es diferenciable, llamada difeología imágen recíproca por $f$. Por ejemplo, si $U \subset S$ y $P(S)$ es una difeología de $S$ entonces $P_{i}(U)$ es la imágen recíproca de la difeología de $S$ por la inclusión $i$ de $U$ en $S$, formada por las placas con valores en $U$.

Dada una colección $\left(S_{i}, P_{i}\left(S_{i}\right)\right)_{i \in I}$ de espacios difeológicos, se llama difeología producto de $\Pi_{i} S_{i}$ a la intersección de las imágenes recíprocas de las proyecciones $\pi_{i}$ en $S_{i}$. Podemos ver que está formado por las placas $p$ tal que $\pi_{i} \circ p \in P_{i}\left(S_{i}\right)$. 
Dada una $C^{k}$ variedad diferenciable $M$, la difeología de variedad $C^{k}$ de $M$ es aquella formada por las $C^{k}$ placas de $M$. En este caso los dos conceptos de diferenciabilidad coinciden.

Se llama $P$-topología de un conjunto $S$ con una difeología $P(S)$ a la topología más fina para la cual cada placa es continua. Observamos que mientras más fina la difeología, menos fina la topología.

Podemos ver que un espacio difeológico $(S, P(S))$ es una n-variedad si y solo si $S$ es localmente difeomorfo a $\mathbf{R}^{n}$ en cada punto, con la P-topología (aquí asumimos que todo abierto de $\mathbf{R}^{n}$ tiene la difeología de variedad $C^{\infty}$ llamada difeología estandar).

Si $k \in \mathbf{N}$, un triplete $(S, P(S), \sim)$ se llama una $C^{k}$-difeología (se llama difeología suave o eds para $k=\infty)$ si $(S, P(S))$ es una difeología y $\sim$ es una colección $\left\{\sim_{F}^{n}, 1 \leq n \leq k, F \in S\right\}$ donde $\sim_{F}^{n}$ es una relación de equivalencia en el conjunto $P_{F}^{n}(S)$ de n-placas $p$ tal que $p(0)=F$ y las relaciones satisfacen una relación de consistencia: $p_{1} \circ \phi \sim_{F}^{n-1} p_{2} \circ \phi$ si $p_{1} \sim_{F}^{n} p_{2}$ y $\phi \in C^{\infty}\left(U^{\prime}, U\right)$ donde $U^{\prime} \subset \mathbf{R}^{m}$ y $p_{i}: U \rightarrow S, i=1,2$.

A la clase de la placa $p(t)$ en $F$ se le denota $[p]$ o $[p]_{F, t}$ cuando sea necesario.

Si además $P_{F}^{n}(S) / \sim_{F}^{n}$ tiene una estructura de espacio vectorial $V_{F}^{n}$ entonces denotamos por $V$ al conjunto

$$
\left\{V_{F}^{n}, F \in S, 1 \leq n \leq k\right\}
$$

y se le llama a $(S, P(S), \sim, V)$ una $C^{k}$ - difeología lineal.

Para una $C^{k}$ difeología $(S, P(S), \sim)$, sea lineal o no, al conjunto $\mathcal{T}_{F}^{n} S:=P_{F}^{n}(S) / \sim_{F}^{n}$ se le llama el $n$ - espacio tangente en $F$ y a la unión disjunta $\mathcal{T}^{n} S:=\bigsqcup_{F \in S} \mathcal{T}_{F}^{n} S$ se le llama el fibrado tangente a $S$.

Sean $(R, P(R), \sim),(S, P(S), \approx)$ dos difeologías suaves. Una función $f: R \rightarrow S$ se llama suave si $f$ es $C^{\infty}$ diferenciable y $f \circ p_{1} \approx_{f(F)}^{n} f \circ p_{2}$ para $p_{1} \sim_{F}^{n} p_{2}$. Al conjunto de tales funciones se le denota por $\mathcal{C}^{\infty}(R, S)$. Si $S=\mathbf{R}$ con la difeología de variedad suave entonces utilizamos la notación $\mathcal{C}^{\infty}(R)$.

Si además $(R, P(R), \sim, V),\left(S, P(S), \approx, V^{\prime}\right)$ son difeologías suaves lineales entonces requerimos que la función

$$
\begin{aligned}
D_{F}^{n} f: \mathcal{T}_{F}^{n} R & \rightarrow \mathcal{T}_{f(F)}^{n} S \\
{[p] } & \mapsto[f \circ p]
\end{aligned}
$$

sea lineal.

Si $f: R \rightarrow S$ y $g: S \rightarrow S^{\prime}$ son suaves entonces $g \circ f$ es diferenciable y preserva las relaciones. Además

$$
\begin{aligned}
D_{F}^{n}(g \circ f)[p] & =[(g \circ f) p] \\
& =\left(D_{f(F)}^{n} g\right)[f(p)] \\
& =\left(D_{f(F)}^{n} g\right)\left(D_{F}^{n} f[p]\right)
\end{aligned}
$$

y ésta es lineal, por lo tanto $g \circ f$ es suave.

Algunos ejemplos son los siguientes 
1. Si $M$ es una variedad diferenciable modelada en un espacio localmente convexo $V$, consideramos $P(M)$ el conjunto de placas suaves (en la definición de variedad). Para cada $F \in M$ escogemos una carta $\left(U_{F}, \alpha_{F}\right)$ alrededor de $F$ y definimos $p_{1} \sim_{F}^{n} p_{2}$ si

$$
D^{m}\left(\alpha_{F} \circ p_{1}\right)(0)=D^{m}\left(\alpha_{F} \circ p_{2}\right)(0) \quad \forall m \leq n .
$$

Definimos $V_{F}^{1}$ a través de la biyección

$$
\begin{aligned}
\mathcal{T}_{F} M & \longrightarrow V \\
{[p] } & \left.\mapsto \frac{d}{d t}\left(\alpha_{F} \circ p\right)(t)\right|_{t=0}
\end{aligned}
$$

2. Si $M$ es una variedad diferenciable y $S=\operatorname{Dif} f_{c}(M)$, definimos $P(S)$ como el conjunto de funciones $p: U \subset \mathbf{R}^{n} \rightarrow S$ tal que $\phi_{p}: U \times M \rightarrow M$ definido por $\phi_{p}(r, m)=$ $p(r) m$ es suave (con respecto a $M$ ). Definimos $p_{1} \sim p_{2}$ con $p_{1}(0)=p_{2}(0)=g \in S$ si

$$
\left.\frac{d^{i}}{d t^{i}}\right|_{t=0} p_{1}(t)(m)=\left.\frac{d^{i}}{d t^{i}}\right|_{t=0} p_{2}(t)(m) \quad \forall m \in M, \forall i \leq s .
$$

Entonces $\mathcal{T}^{1} S=\Gamma_{c}(M)$.

3. En $S=\mathbf{R}^{3} \backslash\{0\}$ consideramos una colección de conos circulares con vértices en el origen, incluyendo el eje $z$. Consideramos la difeología $P_{1}(S)$ formada por las 1placas contenidas a la vez en un solo cono y en un plano perpendicular al plano $x y$ que pasa por el origen, y la difeología $P_{2}(S)$ formada por 2-placas y 1-placas contenidas en un cono. La difeología $P_{1}(S)$ es lineal, definiendo $\sim$ como en [1] y los espacios tangentes son 1-dimensionales. La $P_{2}(S)$ también es lineal, los planos tangentes son 2-dimensionales, excepto en el eje $z$ donde son 1-dimensionales. Si agregamos el origen entonces no son lineales.

Si $(S, P(S), \sim, V)$ es un e.d.s. y $R \subset S$, consideramos la difeología de subespacio $P(R)$ y restringimos $\sim$ a $P(R)$. Entonces $R$ se llama un sub-eds si $P^{n}(R) / \sim_{F}^{n}$ es un subespacio de $P^{n}(S) / \sim_{F}^{n}$ para cada $F \in R$. Por ejemplo, cada componente conexa es un sub-eds.

\section{La difeología tangente}

Si $(S, P(S), \sim, V)$ es un eds, en el espacio $\mathcal{T}^{m} S$ consideramos las placas de la forma

$$
\widetilde{p}\left(r_{1}, \ldots, r_{n}\right)=\left[p\left(r_{1}, \ldots, r_{n}, s_{1}, \ldots, s_{m}\right)\right]_{\left(s_{1}, \ldots, s_{m}\right)}
$$

donde $p$ es una $(\mathrm{n}+\mathrm{m})$-placa y definimos $P\left(\mathcal{T}^{m} S\right)$ como la difeología generada por estas placas.

Sea $[\alpha] \in \mathcal{T}^{m} S, \alpha(0)=F$. Sean $\widetilde{p}_{1}, \widetilde{p}_{2}$ dos $n$-placas en $\mathcal{T}^{m} S$, en $\widetilde{p}_{1}(0)=\widetilde{p}_{2}(0)=\alpha$. Definimos

$$
\widetilde{p}_{1} \approx_{[\alpha]}^{n} \widetilde{p}_{2} \Longleftrightarrow \widetilde{p}_{1}(0) \sim_{F}^{n+m} \widetilde{p}_{2}
$$


Sea $\approx$ el conjunto de tales relaciones. Definimos las operaciones

$$
c\left[\widetilde{p}_{1}\right]^{\mathcal{T}^{m} X}:=\left[\widetilde{c p_{1}}\right]^{\mathcal{T}^{m} X}
$$

donde $c p_{1} \in c\left[p_{1}\right]^{X}$. Y

$$
\left.\left[\widetilde{p}_{1}\right]_{[\alpha]}^{\mathcal{T}^{m} X}+\left[\widetilde{p}_{2}\right]_{[\alpha]}^{\mathcal{T}^{m} X}=\widetilde{\left[p_{1}+p_{2}\right.}\right]_{[\alpha]}^{\mathcal{T}^{m} X}
$$

para $p_{1}+p_{2} \in\left[p_{1}+p_{2}\right]_{F}^{X}$.

Esto define el conjunto $V$ de espacios vectoriales

$$
\mathcal{T}_{[\alpha]}^{n} \mathcal{T}^{m} S:=P^{n}\left(\mathcal{T}^{m} S\right) / \approx_{[\alpha]}^{n}
$$

En [9] se demuestra que $\left(\mathcal{T}^{m} S, P, \approx, V\right)$ es una difeología lineal suave. Además, si $f: S \rightarrow \mathbf{R}$ es suave entonces $D^{m} f: \mathcal{T}^{m} S \rightarrow \mathcal{T}^{m} R$ es suave para todo $m$. Se demuestra también que $\pi: \mathcal{T}^{m} S \rightarrow S$ definido por $\pi\left([\alpha]_{F}\right)=F$ es suave.

Se dice que un eds satisface la condición de contacto si para todo par $p_{1}, p_{2}$ de $(n+1)$ placas definidas en $U=U_{1} \times U_{2}$ donde $U_{1}, U_{2}$ son conjuntos abiertos en $\mathbf{R}^{n}, \mathbf{R}$ respectivamente alrededor del origen, tal que $p_{1}(t, 0)=p_{2}(t, 0)$ para todo $t \in U_{1}$ existe una $(n+1)$-placa $p_{12}$ definida en un vecindario del origen tal que

$$
\left[p_{12}(t, s)\right]_{s}=\left[p_{1}(t, s)\right]_{s}+\left[p_{2}(t, s)\right]_{s}
$$

para todo t. $\mathrm{Y}$ decimos que satisface la condición débil de contacto si la condición se restringe sólo a pares de placas $p_{1}, p_{2}$ tal que $p_{1}(t, 0)=p_{2}(t, 0)=F_{0}$ para todo $t$ y se pide que $p_{12}(t, 0)=F_{0}$ para todo $t$.

Se demuestra en [9] que si $S$ satisface la condición débil de contacto entonces $\mathcal{T}_{F}^{n} S$ es un eds lineal para todo n (un sub-eds lineal de $\mathcal{T}^{n} S$ ), y además, si $f: S \rightarrow R$ es suave entonces

$$
D_{F_{0}}^{m}: \mathcal{T}_{F_{0}}^{n} S \rightarrow \mathcal{T}_{f\left(F_{0}\right)}^{n} R
$$

es suave para todo $n$. Una sección suave del fibrado tangente se llama un campo vectorial y el conjunto de ellos se denota $\Gamma(S)$. Si $\xi \in \Gamma(S)$ y $f \in \mathcal{C}^{\infty}(S)$, se define

$$
\left.\xi(f):=\left.\frac{d}{d t}\right|_{t=0} f(\xi(F)) t\right)
$$

Se demuestra que $\xi$ es una derivación en $\mathcal{C}^{\infty}(S)$ y que $\Gamma(S)$ es un $\mathcal{C}^{\infty}(S)$-módulo.

Si $R, S$ son espacios difeológicos, $\xi \in \Gamma(S)$ y $\alpha: R \rightarrow S$ es un difeomorfismo, entonces $\alpha_{*} \xi$ es el campo vectorial

$$
\alpha_{*} \xi(F):=D_{\alpha^{-1}(F)} \alpha\left(\xi\left(\alpha^{-1}(F)\right)\right)
$$

Sea $\xi_{1}, \xi_{2} \in \Gamma(S)$, entonces $B\left(\xi_{1}, \xi_{2}\right)$ definido por

$$
B\left(\xi_{1}, \xi_{2}\right)(f):=\xi_{1} \xi_{2} f-\xi_{2} \xi_{1} f
$$

es un elemento de $\operatorname{Der}\left(\mathcal{C}^{\infty}(X)\right)$. Sea $\mathcal{M}$ un álgebra maximal de $\Gamma(S)$ con este producto. Para cada subálgebra de este tipo definimos una n-forma como una sección $\omega$ de $\wedge^{n}(\mathcal{T}(S))$ 
tal que $\omega\left(\xi_{1}, \ldots, \xi_{n}\right)(F):=\omega(F)\left(\xi_{1}(F), \ldots, \xi_{n}(F)\right)$ es suave cuando $\xi_{i} \in \mathcal{M}, \forall i=1, \ldots, n$. El conjunto de ellas se denota por $\wedge^{n}(S)$, el cual es un $\mathcal{C}^{\infty}(S)$ módulo y un álgebra asociativa con la operación:

$$
\omega \wedge \eta:=\frac{(k+l) !}{k ! l !} A l t(\omega \otimes \eta)
$$

Definimos $d_{n}: \bigwedge^{n}(X) \rightarrow \bigwedge^{n+1}(X)$ de la siguiente forma:

$$
\begin{aligned}
d_{n} \omega\left(\xi_{1}, \ldots, \xi_{n+1}\right)= & \sum_{i=1}^{n+1}(-1)^{i+1} \xi_{i} \omega\left(\xi_{1}, \ldots, \widehat{\xi}_{i}, \ldots, \xi_{n+1}\right) \\
& +\sum_{i<j}(-1)^{i+j} \omega\left(B\left(\xi_{i}, \xi_{j}\right), \xi_{1}, \ldots, \widehat{\xi}_{i}, \ldots, \widehat{\xi}_{j}, \ldots, \xi_{n+1}\right) .
\end{aligned}
$$

Por ejemplo, si $\omega=\sum_{i \in I} f_{i} d f_{i_{1}} \wedge \cdots \wedge d f_{i_{n}}$ entonces $d \omega=\sum_{i \in I} d f_{i} \wedge d f_{i_{1}} \wedge \cdots \wedge d f_{i_{n}}$.

Se demuestra que $d_{n+1} \circ d_{n}=0$ por lo que podemos definir $Z_{n}(X, \mathbf{R}):=\operatorname{ker}\left(d_{n}\right)$, $B_{n}(X, \mathbf{R}):=\operatorname{Im}\left(d_{n-1}\right) \mathrm{y}$

$$
H_{\mathrm{dR}}^{n}(X, \mathbf{R}):=Z_{n} / B_{n}
$$

llamado el $n$-ésimo grupo de cohomología de $X$.

\section{Precuantización en difeologías suaves}

Una difeología simpléctica es un eds $(S, P(S), \sim, V)$ con una 2-forma cerrada $\Phi$ tal que $\Upsilon: \Gamma(S) \rightarrow \wedge^{1}(S)$ definido por $\Upsilon(\xi)=i(\xi) \Phi$ es $1-1$.

Un campo vectorial $\xi$ se llama Hamiltoniano si existe $f$ tal que $i(\xi) \Phi=d f$. Denotamos por $\operatorname{Ham}(S)$ al conjunto de ellos. Podemos ver que $\operatorname{Ham}(S)$ es un álgebra de Lie: definimos

$$
\mathcal{L}_{\xi} \Phi\left(\xi_{1}, \cdots, \xi_{k}\right)=\xi\left(\Phi\left(\xi_{1}, \cdots, \xi_{k}\right)\right)-\sum_{j=1}^{k} \Phi\left(\xi_{1}, \cdots,\left[\xi, \xi_{j}\right], \cdots, \xi_{k}\right)
$$

entonces

$$
\mathcal{L}_{\xi} \Phi=d \circ i_{\xi} \Phi+i_{\xi} \circ d \Phi
$$

Por tanto si $\Phi$ es simpléctica entonces $\mathcal{L}_{\xi} \Phi=0$. Con la definición de $\mathcal{L}_{\xi} \Phi$ se verifica que

$$
i_{[\xi, \chi]} \Phi=\mathcal{L}_{\xi}\left(i_{\chi} \Phi\right)-i_{\chi} \mathcal{L}_{\xi} \Phi
$$

por tanto para $\Phi$ simpléctica se tiene que

$$
i_{[\xi, \chi]} \Phi=\mathcal{L}_{\xi} i_{\chi} \Phi=\left(d \circ i_{\xi}\right)\left(i_{\chi} \Phi\right)
$$

y por tanto $\operatorname{Ham}(S)$ es una subálgebra de Lie de $\mathcal{M}$.

Con el cálculo anterior se comprueba que el espacio $\operatorname{Ham}_{L}(S)$ de campos $\xi$ tal que $d i_{\xi} \Phi=0$ es también una subálgebra de Lie de $\mathcal{M}$. 
Una función $f \in \mathcal{C}^{\infty}(S)$ se llama Hamiltoniana si $d f=i\left(\xi_{f}\right) \Phi$ para algún $\xi \in \operatorname{Ham}(S)$, y al conjunto de ellas lo denotamos $\mathcal{C}_{H}^{\infty}(S)$. En este espacio definimos

$$
\mathcal{P}(f, g)=\Phi\left(\xi_{f}, \xi_{g}\right)
$$

Vamos a demostrar que este espacio de funciones es un álgebra de Poisson: tenemos que $\Phi\left(\xi_{f}, \chi\right)=\chi f$ y por tanto $\xi_{f} g=-\mathcal{P}(f, g)$.

De la definición de $d \Phi$ tenemos

$$
\begin{gathered}
\xi(\Phi(\chi, \pi))+\chi(\Phi(\pi, \xi))+\pi(\Phi(\xi, \chi)) \\
-\Phi([\xi, \chi], \pi)-\Phi([\chi, \pi], \xi)-\Phi([\pi, \xi], \chi)=0
\end{gathered}
$$

Luego

$$
\begin{array}{r}
\left.\xi_{f} \xi_{h} g\right)+\xi_{g}\left(\xi_{g}\left(\xi_{f}\right) h\right)+\xi_{h}\left(\xi_{g} f\right) \\
+\left[\xi_{f}, \xi_{g}\right] h+\left[\xi_{g}, \xi_{h}\right] h+\left[\xi_{h}, \xi_{f}\right] f=0
\end{array}
$$

Como

$$
\mathcal{P}(f, \mathcal{P}(g, h))=\Phi\left(\xi_{f}, \xi_{\mathcal{P}(g, h)}\right)=\xi_{f} \mathcal{P}(g, h) \xi_{f} \Phi\left(x i_{g}, \xi_{h}\right)
$$

entonces

$$
\mathcal{P}(f, \mathcal{P}(g, h))+\mathcal{P}(g, \mathcal{P}(h, f))+\mathcal{P}(h, \mathcal{P}(f, g))=0
$$

Esto demuestra que $\mathcal{C}_{H}^{\infty}(S)$ es un álgebra de Lie.

De la igualdad

$$
\begin{aligned}
\mathcal{P}(f, g h) & =\Phi\left(\xi_{f}, \xi_{g h}\right) \\
& =\xi_{f}(g h) \\
& =\xi_{f}(g) h+g \xi_{f}(h) \\
& =\mathcal{P}(f, g) h+g \mathcal{P}(f, h)
\end{aligned}
$$

se obtiene que $\left(\mathcal{C}_{H}^{\infty}(S, \mathcal{P})\right)$ es un álgebra de Poisson.

Si $\Phi=d \rho$ entonces definimos para todo $f \in \mathcal{C}_{H}^{\infty}(S)$ el operador

$$
\mathcal{Q}(f)=i \hbar \xi_{f}+\rho\left(\xi_{f}\right)+f
$$

Observamos que $\mathcal{Q}$ es lineal y que $\mathcal{Q}(1)$ es la identidad. Además

$$
\begin{aligned}
\frac{1}{\hbar}[\mathcal{Q}(f), \mathcal{Q}(g)]= & i \hbar\left[\xi_{f}, \xi_{g}\right] \\
& +\xi_{f}\left(\rho\left(\xi_{g}\right)+g\right)-\xi_{g}\left(\rho\left(\xi_{f}\right)+f\right) \\
= & i \hbar\left[\xi_{f}, \xi_{g}\right]+\rho\left(\xi_{\mathcal{P}(f, g)}\right)+\mathcal{P}(f, g) \\
= & i \hbar\left[\xi_{f}, \xi_{g}\right]+\xi_{f}\left(\rho\left(\xi_{g}\right)\right)-\xi_{g}\left(\rho\left(\xi_{f}\right)\right) \\
& -\rho\left(\left[\xi_{f}, \xi_{g}\right]\right)+\rho\left(\left[\xi_{f}, \xi_{g}\right]\right) \\
= & i \hbar\left[\xi_{f}, \xi_{g}\right]+\rho\left(\xi_{\mathcal{P}(f, g)}\right)+d \rho\left(\xi_{f}, \xi_{g}\right) \\
= & \mathcal{Q}(\mathcal{P}(f, g))
\end{aligned}
$$

De esta forma obtenemos que el cuadro de precuantización se extiende a una difeología simpléctica para el caso en que $\Phi$ es exacta. 


\section{Referencias}

[1] Adams, M.; Ratiu, T.; Schmid, R. (1985) "A Lie group structure of diffeomorphism groups and invertible Fourier integral operators, with applications", MSRI Publications, V. Kac, ed., Springer, New York.

[2] Donato, P. (1988) "Géométrie des Orbites Coadjointes des Groupes de Di fféomorphismes", in Lect. Notes in Math. 1416, Springer-Verlag, Berlin: 84-104.

[3] Donato, P. (1992) "Les difféomorphismes du cercle comme orbite symplectique dans les moments de Virasoro", CPT Preprint, CNRS-Luminy.

[4] Donato, P.; Iglesias, P. (1987) "Cohomologie des formes dans les espaces difféologiques", CPT Preprint, CNRS-Luminy.

[5] Iglesias, P. (1985) Fibrations Difféologiques et Homotopie. Thèse de 3-ème cycle, CPT, Marseille.

[6] Kirillov, A.A. (1976) Elements of the Theory of Representations. Springer-Verlag, Berlin.

[7] Souriau, J.M. (1985) "Un algoritme générateur de structures quantiques", Asterisque: 341-399.

[8] Torre, C.A. (1993) "A symplectic structure on coadjoint orbits of diffeomorphism subgroups", Ciencia y Tecnología 17(2): 1-14.

[9] Torre, C.A. (1996) "A tangent bundle on diffeological spaces", Preprint.

[10] Woodhouse, N. (1992) Geometric Quantization. Clarendon Press. 